\title{
Improving effects of mesenchymal stem cells on symptoms of chronic obstructive pulmonary disease
}

\author{
Karaoz $\mathrm{E}^{1,2,3}$, Kalemci $\mathrm{S}^{4}$, Ece $\mathrm{F}^{5,6}$ \\ Istinye University, Faculty of Medicine, Department of Histology\&Embryology, Istanbul, Turkey. \\ skalemci79@gmail.com
}

\begin{abstract}
AIM: We aimed to identify the improving effects of umbilical cord tissue-derived (UCTD) MSCs on the symptoms of COPD in our phase 1.2 clinical study.

MATERIAL METHODS: Our study consisted of five patients with COPD. Respiratory function tests, SGRQ symptom, activity and impact scores and 6-minute walk test (6MWT) were examined before UCTD MSC treatment. All the patients were administered a total of 4 doses of UCTD MSCs by intravenous infusion at two-week intervals. All the tests were repeated three months after the treatment for evaluation of the response to MSCs treatment.

RESULTS: The mean age of five male patients was 56. The mean pretreatment FEV1/FVC ratios were 66.9 $\%$. Pretreatment mean SGRQ symptom, activity and impact scores of the patients were $78.2,83.8$ and 58.02 respectively. The mean walking distance of the patients was 307 meters before MSCs treatment. The mean FEV1/FVC value of raised to $69.58 \%$ after the treatment. The mean SGRQ symptom, activity and impact scores were noted as $39.8,60.98$ and 45.18 respectively. The mean walking distance of the patients raised to 362 meters after the treatment.

CONCLUSIONS: Our results showed that four doses of MSC treatment considerably alleviated the severity of symptoms of COPD (Tab. 2, Fig. 7, Ref. 25). Text in PDF www.elis.sk.

KEY WORDS: disease progression, mesenchymal stromal cells, chronic obstructive pulmonary disease, umbilical cord tissue.
\end{abstract}

\section{Introduction}

Inflammation and emphysematous destruction is commonly accompanied with COPD. It is estimated that COPD affects 174.5 million people in the world and carries a high morbidity and mortality risk. There is no therapy that can regenerate destroyed alveoli (1-3).

MSCs are multipotent, non-hematopoietic progenitors and are able to differentiate into many different cell lineages. Additionally, they have the capacity for self-renewal. Many authors reported that MSCs can be readily harvested from several tissues and expanded with high efficiency and displays strong immunosuppressive immunomodulatory and neuroprotective properties (4). The benefit of MSCs has been shown in many pulmonary conditions such as: asthma, COPD, emphysema and acute respiratory distress syndrome in many preclinical studies (5-7). However, further clini-

${ }^{1}$ Istinye University, Faculty of Medicine, Department of Histology\& Embryology, Istanbul, Turkey, ${ }^{2}$ Istinye University, Center for stem Cell andTissue Engineering Research\&Practice, Istanbul, Turkey, ${ }^{3}$ Liv Hospital, Center for Regenerative Medicine and Stem Cell Manufacturing (LivMedCell), Istanbul, Turkey, ${ }^{4}$ Department of Chest Diseases, Medical Park Gebze Hospital, Kocaeli, Turkey, ${ }^{5}$ Department of Chest Diseases, Liv Hospital, Istanbul, Turkey, and ${ }^{6}$ Istinye University, Faculty of Medicine, Department of Chest Diseases, Istanbul, Turkey

Address for correspondence: S. Kalemci, Medical Park Gebze Hospital, 41400, Gebze, Kocaeli, Turkey.

Phone: +90.5079845193 cal studies focused on the efficacy of MSCs on the treatment of COPD are needed (8-10).

Our clinical study was aimed to investigate the effects of MSCs derived from umbilical cord-derived on the treatment of five patients with COPD and to review similar studies in literature.

\section{Material and methods}

Our early phase clinical study was designed as an open-label, single-armed and early phase (1/2) study carried out in LIV hospital in Istanbul and included 5 cases suffering from COPD. The study protocol was approved by the decision on 22nd July 2015 and 2015-13/01 number of ethics committee of clinical research of Bahcesehir University. Informed consent form including the knowledge about the risks of the study was obtained from all the patients. The diagnosis of COPD was confirmed with the criteria of Global Initiative for Chronic Obstructive Lung Disease (GOLD). Pulmonary function tests were performed by the same technician using the Jaeger MasterScreenPneumo V452I device (Care Fusion, Hochberg, Germany). The best test result of the 3 consecutive measurements was recorded. Forced expiratory volume in 1 second, forced vital capacity (FVC), and percentage of FVC expelled in the first second of forced expiration were evaluated according to the American Thoracic Society guidelines.

The St. George's Respiratory Questionnaire (SGRQ) symptom, activity and impact scores were used to quantify the impact 
of COPD on symptoms of the patients. Before MSCs treatment, a six-minute walk test was used for identifying the functional status of our patients. The results of the six-minute walk tests were also used to evaluate the response to treatment after MSCs treatment.

Patients with coexisting diseases such as: extrapulmonary infection, psychosocial problems, active smoking, drug/alcohol abuse, cancer, immunosuppressive disease, pregnancy, active hepatitis, active renal disease, and serious cardiopulmonary diseases were excluded from the study.

After the pretreatment measurements, all the patients were administered a total of 4 doses of umbilical cord tissue-derived (UCTD) MSCs (1-2x106BM-MSCs $/ \mathrm{kg}$ ) by intravenous infusion at two-week intervals. Respiratory function tests, SGRQ scores, and six-minute walking tests were repeated and recorded in all patients three months after the treatment. Umbilical cord tissuederived MSCs, which were manufactured under cGMP (current Good Manufacturing Practice) conditions (LivMedCellIstanbul, Turkey) were used in this study. Cell preparation steps were performed according to cGMP requirements as described previously $(11,12)$. The cells were characterized by identifying the potential for differentiation using a flow cytometer and immunohistochemical analysis, cell aging, cell cycle, annexin V/PI and telomerase enzyme activity at the third passage. The quality control and quality assurance for the production of these cells were conducted in accordance with the standards of the Turkey Pharmaceuticals and Medical Devices Agency (TMMDA) (13).

\section{Results}

All the patients were male and the mean age of the patients was 56 (range from 48 to 73) years. The mean FEV1/FVC value of five patients was $66.9 \%$ before MSCs treatment. The mean FEV1/FVC value of raised to $69.58 \%$ after treatment. Pre and post-treatment respiratory function results were detailed in Table 1.

Pretreatment mean SGRQ symptom, activity and impact scores of the patients were $78.2,83.8$ and 58.02 respectively. We observed significant improvements in SGRQ symptom activity and

Tab. 1. The results of the pre and posttreatment pulmonary function tests of the cases.

\begin{tabular}{lccc}
\hline Age and Gender & FEV1 $(\mathrm{ml})$ & FVC $(\mathrm{ml})$ & FEV1/FVC (\%) \\
\hline $48 \mathrm{y}$, male & $3.10 / 3.12$ & $4.49 / 4.22$ & $69 / 74$ \\
$51 \mathrm{y}$, male & $1.02 / 1.27$ & $1.55 / 1.82$ & \\
$68 \mathrm{y}$, male & $3.44 / 3.65$ & $4.99 / 5.05$ & $69 / 73$ \\
$40 \mathrm{y}$, male & $0.56 / 0.60$ & $0.87 / 0.98$ & $64.5 / 60.9$ \\
$73 \mathrm{y}$, male & $1.05 / 1.20$ & $1.60 / 1.70$ & $66 / 71$ \\
\hline
\end{tabular}

Tab. 2. Pre and posttreatment SGRQ scores and six-minute walking test results of the cases.

\begin{tabular}{lcccc}
\hline SGRQ scores & Symptom & Activity & Impact & $\begin{array}{c}\text { Six-minute } \\
\text { walking (meter) }\end{array}$ \\
\hline 48y, male & $91 / 62$ & $79 / 48$ & $61 / 58$ & $355 / 390$ \\
51y, male & $64 / 23$ & $94 / 63$ & $78 / 46$ & $266 / 290$ \\
$68 y$, male & $68 / 43$ & $66 / 40$ & $31 / 12$ & $374 / 536$ \\
40y, male & $97 / 56$ & $92 / 79$ & $54 / 50$ & $280 / 312$ \\
$73 y$, male & $71 / 15$ & $88 / 75$ & $66 / 60$ & \\
\hline
\end{tabular}

Pre and post treatment FEV1 values of the patients

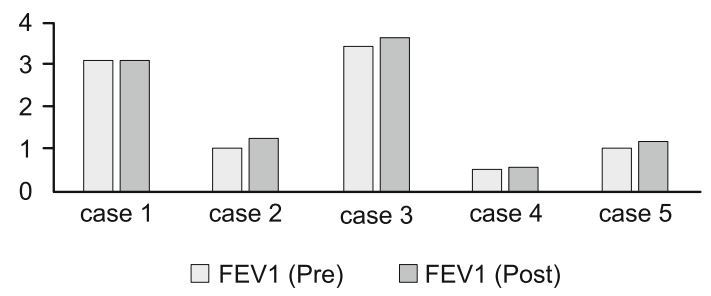

Fig. 1. Comparisons of pre and post-treatment FEV1 values of the cases.

Pre and post treatment FVC values of the cases

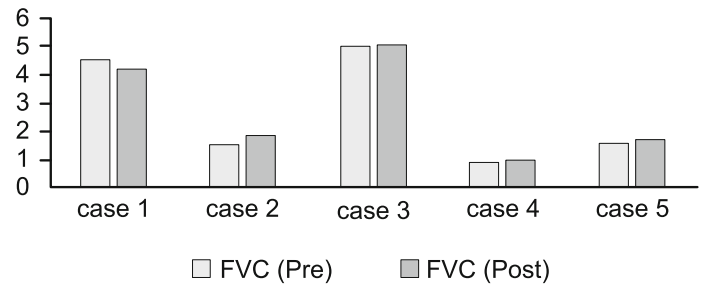

Fig. 2. Comparisons of pre and post-treatment FVC values of the cases.

Pre and post treatment FEV1/FVC values of the cases

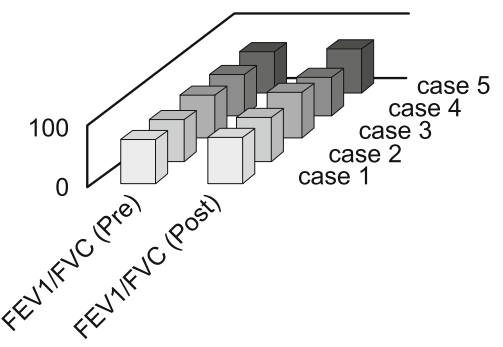

Fig. 3. Comparisons of pre and post-treatment FEV1/FVC values of the cases.

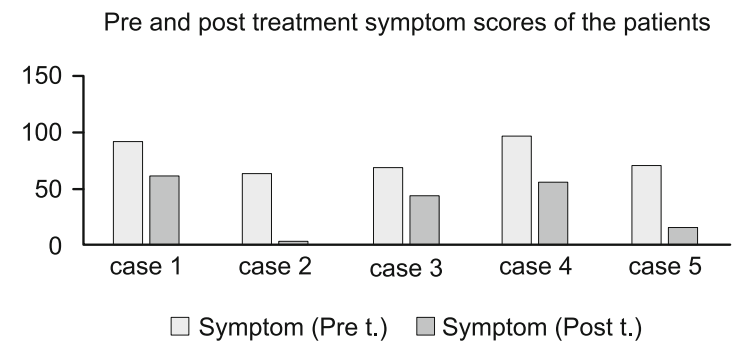

Fig. 4. Comparisons of pre and post-treatment SGRQ symptom scores of the cases.

impact scores of the patients after treatment. Post-treatment mean SGRQ symptom, activity and impact scores were noted as 39.8 , 60.98 and 45.18 respectively. According to the results of the sixminute walk test, the mean walking distance of the patients was 307 meters before MSCs treatment whereas, after the treatment, the mean walking distance of the patients raised to 362 meters. Pre and post-treatment SGRQ and six-minute walk test results were detailed in Table 2. 
Pre and post treatment activity scores of the cases

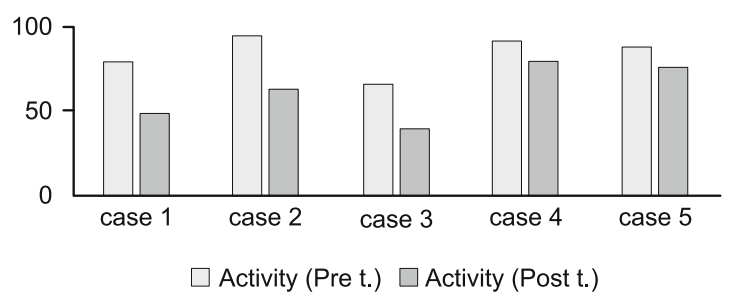

Fig. 5. Comparisons of pre and post-treatment SGRQ activity scores of the cases.

Pre and post treatment impact scores of the patients

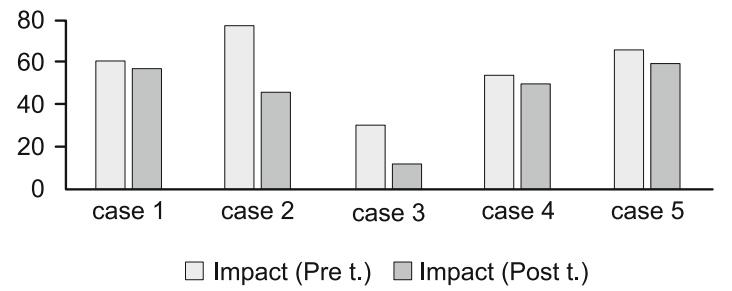

Fig. 6. Comparisons of pre and post treatment SGRQ activity scores of the cases.

Pre and post treatment 6 min. walking test result of the cases

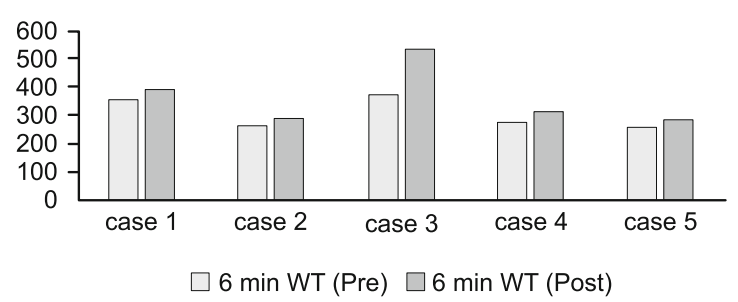

Fig. 7. Pre and post-treatment six-minute walking test results of the cases.

Comparisons of pretreatment and posttreatment FEV1, FVC and FEV1/FVC values of the cases were shown in Figures 1, 2 and 3 respectively. Comparisons of pretreatment and posttreatment SGRQ symptom, activity and impact scores of the cases were shown in Figures 4, 5 and 6. Comparisons of the six-minute walk test results of the cases was shown in Figure7.

\section{Discussion}

Recent studies focused on the new therapeutic agents, which may prevent oxidative damage and anti-protease activities $(3,8$, $10,14)$. Dennis et al. reported that MSCs are multipotent cells that play a pivotal role in various stages of lung development and lung homeostasis and potentially lung regeneration. MSCs have also anti-inflammatory and paracrine properties and are promising as a cell-based treatment strategy for the repair of damaged alveolar tissue in emphysema $(2,8,14,15)$. Previous studies showed that intravenous infused MCSs could migrate to the site of injury to repair the damaged tissue. However, the mechanism for MSC mi- gration is still unclear. It seems to be related with the chemotaxis migration due to hypoxia $(16,17)$.

One of the striking features of mesenchymal stem cells is their activity on different components of the immune response such as macrophages. MSCs are multipotent fibroblast-like cells and have in vivo functions such as: contribution to tissue maintenance and regeneration and modulation of immune responses. Intact macrophage activation and function are essential for the resolution of inflammation, subsequent regenerative procedures and returning to hemostasis, particularly in COPD. M1 and M2b subtypes exhibit a weak phagocytic activity whereas M2a has a stronger phagocytic activity. In the presence of MSCs, yeast ingestion by M1 and M2b increases while it decreases for M2a macrophages. It has been reported that MSCs promote macrophage polarization toward M2b macrophages. Peng et al. also showed that intravenous human umbilical cord blood MSCs could reduce inflammatory response by stimulating M1/M2 subtype conversion in macrophages of mice $(14,15)$.

Systematic review and meta-analysis about preclinical studies focused on the therapeutic effects of stem cell administration in patients with COPD defined the mechanisms of MSCs role in preclinical COPD treatment as attenuating airway inflammation as well as stimulating lung tissue repair (18). In the lights of preclinical findings, a multicenter clinical trial of MSC administration in the treatment of patients with COPD had been carried out, but the results in human studies were reported as less promising compared to the findings of preclinical studies (18). Few human studies that investigated the role of MSCs in the treatment of patients with COPD reported short- term safety, but displayed no convincing clinical benefits. The lack of beneficial effects on clinical outcomes could be attributed to factors such as: source of MSC, administration route, dosage and dose intervals (15).

Bone marrow (BM) is a main source for the isolation of multipotent MSCs, harvesting procedure is highly invasive and the number, differentiation potential and maximal life span of MSCs obtained from BM decline with increasing age (19). Thus, alternative MSCs sources were subjected to intensive research. Currently, the umbilical cord MSCs include cells derived from the total umbilical cord or its different sections (21). Umbilical cord tissuederived (UCTD) MSCs were used in this Phase 1, 2 clinical study.

Improvements in the respiratory function tests, SGRQ symptom activity, and impact scores were used to evaluate the outcomes of UCTD MSC treatment in our patients with COPD. Spirometry is defined as the gold standard for the diagnosis of COPD and consists of 1) forced vital capacity (FVC), 2) forced expiratory volume in one second (FEV1) and 3) FEV1/FVC ratio. A diagnosis of COPD is confirmed by spirometry, when the FEV1/FVC ratio is $<0.70$ (22). FEV1/FVC ratios of our patients were considerably lower than 0.70 before treatment. After four doses of MSCs treatment, FEV1/FVC ratios of the patients raised to normal levels.

High scores of SGQR indicate a bad performance status (23). According to the results of SGRQ, symptom, activity and impact scores of our patients with COPD were considerably higher before MSCs treatment. Pre-treatment values of these scores were consistent with the severe symptoms of COPD in our patients. After 
MSCs treatment, a decrease in the values of symptoms, activity and impact scores of our patients was evident.

The presence of factors such as: reduced exercise capacity and quality of life are closely related to the mortality in patients with COPD. An assessment of functional capacity is very important in terms of fully understanding of pathogenesis. An objective evaluation of functional exercise capacity is carried out by using various methods. The 6-minute walk test (6MWT) is widely used to assess the functional status of patients with COPD. One of the indications for the 6MWT is the evaluation of the response to medical interventions in patients with COPD (24-26). We preferred 6MWT for the assessment of functional exercise capacity in patients before treatment. According to the results of 6MWT, functional exercise capacities of our patients were significantly lower before MSCs treatment. Improvements in the results of 6MWT in our patients showed us that MSCs treatment was considerably effective in terms of the correction of a reduced functional exercise capacity.

\section{Conclusions}

Our phase $1 / 2$ clinical study showed that a total of 4 doses of umbilical cord tissue-derived (UCTD) MSC treatment might be helpful for alleviating the severity of symptoms in patients with COPD. Additionally, respiratory function tests, SGRQ symptom, activity and impact scores, and $6 \mathrm{MWT}$ are effective tools for the pretreatment evaluation of the severity of symptoms in patients with COPD. They are also effective for the evaluation of the response to MSC treatment. Of course, further prospective clinical research is required to identify the optimal source of MSCs, best route to administer MSCs, optimal dose, and intervals in treatment of patients with COPD.

\section{References}

1. Coppolino I, Ruggeri P, Nucera F, Cannavò MF, Adcock I, Girbino G, Caramori G. Role of Stem Cells in the Pathogenesis of Chronic Obstructive Pulmonary Disease and Pulmonary Emphysema. COPD J Chronic Obstr Pulm Dis 2018; 15 (5): 536-556.

2. Gu W, Song L, Li XM, Wang D, Guo XJ, Xu WG. Mesenchymal stem cells alleviate airway inflammation and emphysema in COPD through downregulation of cyclooxygenase-2 via p38 and ERK MAPK pathways. Sci Rep 2015; 5 (1): 8733.

3. Kim YS, Kokturk N, Kim JY, Lee SW, Lim J, Jin Choi S, Oh W et al. Gene Profiles in a Smoke-Induced COPD Mouse Lung Model Following Treatment with Mesenchymal Stem Cells Mol Cells 2016; 39: 728-733.

4. Kabatas S, Demir CS, Civelek E, Yilmaz I, Kircelli A, Yilmaz C, Akyuva $\mathbf{Y}$ et al. Neuronal regeneration in injured rat spinal cord after human dental pulp derived neural crest stem cell transplantation. Bratisl Lek Listy 2018; 119 (3): 143-151.

5. Antunes MA, Abreu SC, Cruz FF, Teixeira AC, Lopes-Pachero M, Bandeira E, Olsen PC et al. Effects of different mesenchymal stromal cell sources and delivery routes in experimental emphysema. Respir Res 2014; 15 (1): 118 6. Cruz FF, Weiss DJ, Rocco PR. Prospects and progress in cell therapy for acute respiratory distress syndrome. Expert Opin Biol Ther 2016; 16 (11): 1353-1360.

7. Lee H, Kim KC, Choi SJ, Hong YM. Optimal dose and timing of umbilical stem cells treatment in pulmonary arterial hypertensive rats. Yonsei Med J 2017; 58 (3): 570-580.
8. Jin Z, Pan X, Zhou K, Bi H, Wang L, Yu L, Wang Q. Biological effects and mechanisms of action of mesenchymal stem cell therapy in chronic obstructive pulmonary disease. J Int Med Res 2015; 43 (3): 303-310.

9. Chang Y, Hee Park S, Huh JW, Lim CM, Koh Y, Hong SB. Intratracheal Administration of Umbilical Cord Blood-Derived Mesenchymal Stem Cells in a Patient with Acute Respiratory Distress Syndrome. J Korean Med Sci 2014; 29: 438-440.

10. Antunes MA, Lapa E Silva JR, Rocco PR. Mesenchymal stromal cell therapy in COPD: from bench to bedside. Int J Chron Obstruct Pulmon Dis 2017; 12: 3017-3027.

11. Dai A, Baspinar O, Yesilyurt A, Sun E, Aydemir CI, Oztel ON, Capkan DU et al. Efficacy of stem cell therapy in ambulatory and nonambulatory children with Duchenne muscular dystrophy - Phase I-II. Degener Neurol Neuromuscul Dis 2018; 8: 63-77.

12. Okur SC, Erdogan S, Demir CS, Gunel G, Karaoz E. The Effect of Umbilical Cord-derived Mesenchymal Stem Cell Transplantation in a Patient with Cerebral Palsy: A Case Report. Int J stem cells 2018 May; 11 (1): 141-147.

13. Kabatas S, Civelek E, Inci C, Yalcinkaya EY, Gunel G, Kir G, Albayrak E et al. Wharton's Jelly-Derived Mesenchymal Stem Cell Transplantation in a Patient with Hypoxic-Ischemic Encephalopathy: A Pilot Study. Cell Transplant 2018 Oct; 27 (10): 1425-1433.

14. D'Agostino B, Sullo N, Siniscalco D, De Angelis A, Rossi F. Mesenchymal stem cell therapy for the treatment of chronic obstructive pulmonary disease. Expert Opin Biol Ther 2010 May; 10 (5): 681-687.

15. Kruk DMLW, Heijink IH, Slebos D-J, Timens W, Ten Hacken NH. Mesenchymal Stromal Cells to Regenerate Emphysema: On the Horizon? Respiration 2018; 96 (2): 148-158.

16. Xu W, Xu R, Li Z, Wang Y, Hu R. Hypoxia changes chemotaxis behaviour of mesenchymal stem cells via HIF-1alpha signalling. J Cell Mol Med 2019; 23 (3): 1899-1907.

17. Hu X, Wu R, Jiang Z, Wang L, Chen P, Zhang L, Yang L et al. Leptin signaling is required for augmented therapeutic properties of mesenchymal stem cells conferred by hypoxia preconditioning. Stem Cells 2014; 32 (10): 2702-2713.

18. Liu X, Fang Q, Kim H. Preclinical Studies of Mesenchymal Stem Cell (MSC) Administration in Chronic Obstructive Pulmonary Disease (COPD): A Systematic Review and Meta-Analysis. PLoS One 2016; 11 (6): e0157099.

19. Arutyunyan I, Elchaninov A, Makarov A, Fatkhudinov T. Umbilical Cord as Prospective Source for Mesenchymal Stem Cell-Based Therapy. Stem Cells Int 2016; 2016: 6901286.

20. Kern S, Eichler H, Stoeve J, Kluter H, Bieback K. Comparative analysis of mesenchymal stem cells from bone marrow, umbilical cord blood, or adipose tissue. Stem Cells 2006; 24 (5): 1294-1301.

21. Bailey KL. The importance of the assessment of pulmonary function in COPD. Med Clin North Am 2012; 96 (4): 745-752.

22. Sciriha A, Lungaro-Mifsud S, Scerri J, Magro R, Camilleri L, Montefort $\mathrm{S}$. Health status of COPD patients undergoing pulmonary rehabilitation: A comparative responsiveness of the CAT and SGRQ. Chron Respir Dis 2017; 14 (4): 352-359.

23. Çiftçi F, Şen E, Yıldız A, Saryal Ö. Does exercise capacity, dyspnea level, or quality of life actually predict mortality in patients with COPD? 8-year follow-up. Tuberk Toraks 2019; 67 (2): 83-91.

24. Celli B, Tetzlaff K, Criner G, Polkey MI, Sciurba F, Casaburi R, TalSinger R et al. The 6-Minute-Walk Distance Test as a Chronic Obstructive Pulmonary Disease Stratification Tool. Insights from the COPD Biomarker Qualification Consortium. Am J Respir Crit Care Med 2016 Dec; 194 (12): 1483-1493. 25. ATS statement: guidelines for the six-minute walk test. Am J Respir Crit Care Med 2002 Jul; 166 (1): 111-117.

Received October 21, 2019. Accepted December 18, 2019. 